\title{
Decision Making in Children with Attention-Deficit/Hyperactivity Disorder
}

\author{
Akira Yasumura1,2*, Yoshiyuki Takimoto3 ${ }^{3}$ Eisuke Nakazawa3 ${ }^{3}$, Masumi Inagaki1 \\ ${ }^{1}$ Department of Developmental Disorders, National Institute of Mental Health, National Center of Neurology \\ and Psychiatry, Tokyo, Japan \\ ${ }^{2}$ Department of Neuropsychiatry, The University of Tokyo Hospital, Tokyo, Japan \\ ${ }^{3}$ Department of Biomedical Ethics, The University of Tokyo, Tokyo, Japan \\ Email: "a-vasumura@umin.ac.jp
}

Received 20 April 2016; accepted 2 June 2016; published 6 June 2016

Copyright (C) 2016 by authors and Scientific Research Publishing Inc.

This work is licensed under the Creative Commons Attribution International License (CC BY). http://creativecommons.org/licenses/by/4.0/

(c) (i) Open Access

\section{Abstract}

Background: Informed consent forms and clinical study participation explanations contain many specialized words including medical terms that are difficult to understand. The difficulty is particularly obvious for children with developmental disorders who show attention or similar problems. This study quantitatively evaluated the decision-making ability of these children using the Wechsler Intelligence Scale for Children-III (WISC-III) as a preliminary study for a multi-faceted investigation that would also use physiological indices. Methods: Participants were 11 children with Attention Deficit/Hyperactivity Disorder (AD/HD). The WISC-III was used for quantitative evaluation of their decision-making ability. Results of intelligence quotients (IQs), group indices, and subtest scores were analyzed. Results: The mean Performance IQ was four points lower than the mean Verbal IQ. The mean score for the Processing Speed index was lower by more than one standard deviation (SD). The mean scores for the Coding and Object Assembly subtests were lower by more than two SDs. Conclusion: The WISC-III results for IQ and group indices suggested the efficacy of auditory explanations. In addition, the subtest results suggested the necessity to pay sufficient attention to risk-benefit weighting in explanations. These findings suggested that the decision-making ability of children with AD/HD could be assessed using the WISC-III.

\section{Keywords}

Attention-Deficit/Hyperactivity Disorder (AD/HD), Decision Making, Informed Consent

\section{Introduction}

Informed Consent (IC) must be obtained from participants or their parents to be enrolled in a clinical study. In a

${ }^{*}$ Corresponding author.

How to cite this paper: Yasumura, A., Takimoto, Y., Nakazawa, E. and Inagaki, M. (2016) Decision Making in Children with Attention-Deficit/Hyperactivity Disorder. Open Journal of Pediatrics, 6, 158-162. 
clinical study on children, informed assent (IA) must be obtained from the child in addition to consent from the parent.

Therefore, the decision-making ability of the participant must be evaluated in advance. However, evaluation of decision-making ability is a challenge when the participants are children with disorders.

Recently, numerous studies have been conducted on developmental disorders; some reported that children with attention deficit/hyperactivity disorder (AD/HD) showed specific behavioral problems including inattentiveness, impulsiveness, and hyperactivity. In addition, previous studies suggested that these children had only restricted rationality with a limited range of cognition/reasoning; thus, their decision-making capacities for IC/ IA may be low [1] [2].

In healthy individuals, cognitive ability does not directly correspond to decision-making ability. However, in patients with the above disorders, evaluation of cognitive ability is expected to provide effective information in determining decision-making ability level.

The Wechsler Intelligence Scale for Children-III (WISC-III) is a representative test for evaluating cognitive ability. Cognitive ability assessed by the WISC-III is defined as the combination of the participant's individual abilities to act intentionally and think rationally in the environment and to deal effectively with it [3].

Some previous case-based studies have investigated cognitive characteristics of children with developmental disorders using the WISC-III intelligence quotients (IQ) or group indices [4] [5]. However, to date, no groupbased study involving the WISC-III subtests has been reported on the quantitative characteristics of decisionmaking ability for IC/IA of children with developmental or mental disorders.

This study investigated decision-making ability for IA quantitatively using the WISC-III subtests.

\section{Materials and Methods}

\subsection{Participants}

The participants were those diagnosed with AD/HD based on the Diagnostic and Statistical Manual of Mental Disorders Fourth Edition (DSM-IV-TR), at the National Center Hospital, Neurology and Psychiatry's pediatric outpatient clinic, who provided written informed consent for study participation after receiving explanations between 2008 and 2013. One patient was taking methylphenidate and atomoxetine.

Sample size was determined based on previous studies in children with AD/HD [1].

The ethics board of the National Center of Neurology and Psychiatry reviewed and approved this study's methods.

\subsection{Methods}

WISC-III intelligence quotients (IQ) and the WISC-III subtest were used. WISC-III mean scores of typically developing children were used as the control to compare the standard deviation. Therefore, the standard value of IQ and the group index was $100(S D=15)$ and the standard value for subtests was $10(S D=1.5)$.

\section{Results}

Age range was $9.64 \pm 2.14$ years. Detailed participant profiles are shown in Table 1 . The mean IQs and group indices of all participants are summarized in Figure 1. The mean scores were $84.8 \pm 19.4$ for Full-scale IQ, 88.3 \pm 18.0 for Verbal IQ, and $84.4 \pm 19.8$ for Performance IQ. The mean Performance IQ was four points lower than the Verbal IQ.

The group indices means were $90.4 \pm 18.3$ for Verbal Comprehension, $86.9 \pm 18.6$ for Perceptual Organization, $86.5 \pm 10.4$ for Freedom from Distractibility, and $84.0 \pm 21.5$ for Processing Speed. In the comparison of the four group indices, the mean Processing Speed IQ was the lowest, or lower than one SD.

The mean subtest scores of all participants are summarized in Figure 2. The scores were $9.2 \pm 3.1$ for Picture Completion, $8.0 \pm 4.1$ for Information, $6.9 \pm 3.4$ for Coding, $8.3 \pm 3.3$ for Similarities, $9.0 \pm 4.6$ for Picture Arrangement, 7.1 \pm 3.4 for Arithmetic, $8.0 \pm 3.7$ for Block Design, $9.1 \pm 4.1$ for Vocabulary, $5.8 \pm 3.1$ for Object Assembly, $8.3 \pm 3.2$ for Comprehension, $7.4 \pm 4.5$ for Symbol Search, $8.4 \pm 2.2$ for Digit Span, and $8.0 \pm 3.0$ for Mazes. In particular, the mean scores for Coding and Object Assembly were lower by more than two SDs. Correlation was analyzed by Pearson's correlational coefficient between the age and the scores of Coding and Object Assembly, respectively. The result suggested that the tendency remained unchanged even in higher ages and 
Table 1. Participant profiles.

\begin{tabular}{ccccccccccccc}
\hline Case & 1 & 2 & 3 & 4 & 5 & 6 & 7 & 8 & 9 & 10 & 11 \\
\hline Age & 7.4 & 11.1 & 8.6 & 9.3 & 7.0 & 8.4 & 11.9 & 14.0 & 10.4 & 10.2 & 7.7 \\
Sex & $\mathrm{M}$ & $\mathrm{M}$ & $\mathrm{M}$ & $\mathrm{M}$ & $\mathrm{M}$ & $\mathrm{F}$ & $\mathrm{M}$ & $\mathrm{M}$ & $\mathrm{M}$ & $\mathrm{M}$ & $\mathrm{M}$ \\
Dominant hand & $\mathrm{R}$ & $\mathrm{R}$ & $\mathrm{R}$ & $\mathrm{R}$ & $\mathrm{R}$ & $\mathrm{R}$ & $\mathrm{R}$ & $\mathrm{R}$ & $\mathrm{R}$ & $\mathrm{L}$ & $\mathrm{R}$ \\
\hline
\end{tabular}

M: Male, F: Female, R: Right, L: Left.

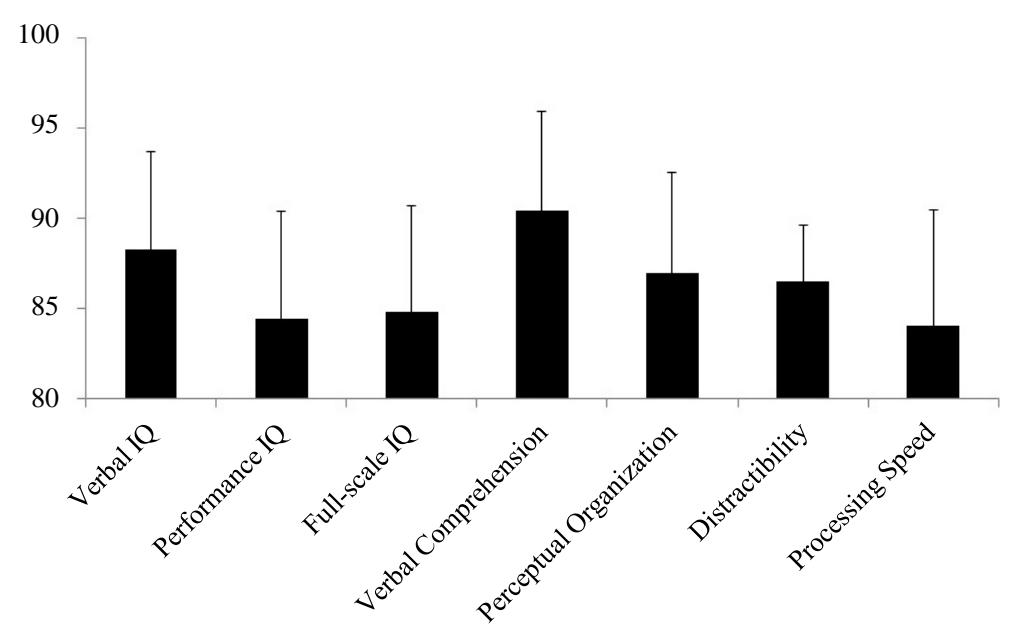

Error bars show standard errors.

Figure 1. Mean IQs and group indices of all participants.

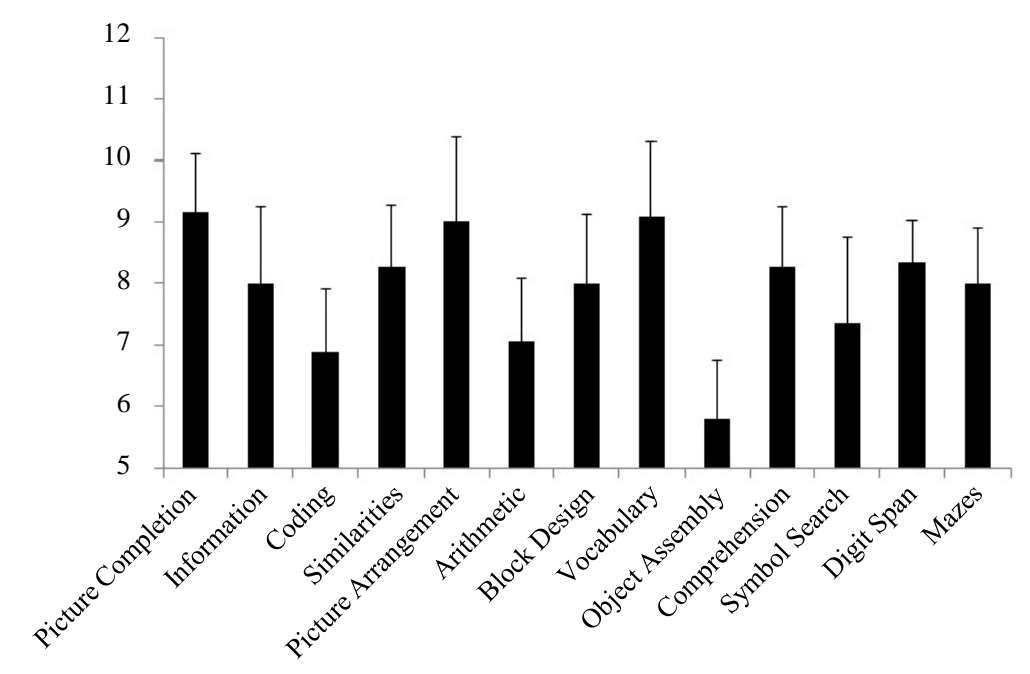

Error bars show standard errors.

Figure 2. Mean subtest scores of all participants.

therefore the difficulty persisted (Coding: $r=-0.528, p=0.095$; Object Assembly: $r=0.343, p=0.302$ ).

\section{Discussion}

This study investigated decision-making ability in children with AD/HD using WISC-III tests. The principal symptoms of children with AD/HD are inattention, hyperactivity, and impulsivity [6], which may suggest their decision-making ability tends to be lower than that of the typically developing children. The tendency may be 
more obvious in understanding written or oral explanation or content, particularly in an unfamiliar situation.

Complex medical terms and other expressions used in informed consent forms sometimes pose a difficulty in understanding. One such example was reported in a clinical study enrolling 287 adult cancer patients; $70 \%$ of the participants did not understand that the test drug had not yet been proven effective [7]. However, another study reported that understanding and satisfaction were improved by shortening or simplifying the texts. Therefore, the participants may be able to understand the explanation sufficiently to give their consent or agreement, if their decision-making ability is validly assessed and IC/AC forms are customized based on the evaluation [8].

\subsection{Characteristics Indicated by IQ Results}

In this study, the average IQ scores were within two SDs for the Full-scale, Verbal, and Performance scores; this indicated that all participants showed normal range of intelligence. However, the mean performance IQ score was lower by four points than that of verbal IQ. Verbal IQ assessed with the WISC-III indicates that the comprehension faculty of verbal information is through the ear; therefore, it is related closely to auditory cognition [9]. It is also an index of the auditory/sound processing faculty closely related to crystallized intelligence including judgment or habits obtained from past learning experiences [4]. On the other hand, Performance IQ is an index for the visual/motor processing faculty closely related to fluid intelligence, facilitating adaptation to a new environment [4]. If these findings are applied to the IA explanation procedure, the superiority of verbal IQ scores observed in this study suggests the effectiveness of auditory support in presenting the IA form. Specifically, reading the text aloud, responding to questions orally, or similar procedures should be effective. While the participants in this study were children with AD/HD, there was a previous study with patients with pervasive developmental disorder reporting Verbal IQ less than Performance IQ as a cognitive characteristic. Therefore, the IA presentation method should be adjusted depending on the type of disorder [10].

\subsection{Characteristics Indicated by Group Index}

An analysis of the group index results showed that the score for Processing Speed was significantly low; this may be closely related to the low Performance IQ score. Processing Speed is related to the ability to process a large volume of visual information correctly without feeling [4]. As suggested in the IQ characteristics analysis, this finding also indicated the efficacy of visual support.

\subsection{Characteristics Indicated by Subtests}

Among the subtest items, scores for Coding and Object Assembly were extremely low. This tendency remains unchanged even in higher ages, suggesting persistent difficulty after the infant stage. Coding is a task under the Processing Speed index that consists of transcribing a geometric figure (Code A) or number (Code B) and the paired simple symbol. The task is supposedly related to abilities including following orders, action agility, speed, and accuracy of administrative work, and visual short-term memory [3] [4]. Low scores for Coding suggest that the participant is poor at monotonous visual tasks and this finding corresponds to a characteristic of individuals with $\mathrm{AD} / \mathrm{HD}$. Therefore, it is not effective to simply show them an explanation document full of text at one time; procedures are necessary to avoid monotony such as reading the text by block, receiving interspersed questions, and confirming the content again after explanation.

Object Assembly is a subtask under the Perceptual Organization index, where pieces are presented in a specific arrangement and the subject is asked to combine the pieces to complete a specific form. It is supposedly related to the abilities to use sensorimotor feedback, forecast correlations among parts, think flexibly, or perceive the whole from the parts [3] [4] [11]. Applying these findings to IA explanation, appropriate risk-benefit weighting of the study, is difficult [2]. Therefore, sufficient explanation should be provided to the participant without underestimating the risk in the study.

\subsection{Limitations}

The study was based on the results of the Japanese version of the WISC-III. The Japanese version of the WISCIV was published in 2010. Therefore, new studies using the more recent version will be required. In addition, a greater number of participants are required for a more detailed study on the impact of factors, including growth. Further studies are also necessary that focus on other disorder groups as well as on relationships with cerebral 
functions.

\section{Acknowledgements}

This study was partially supported by the Grant-in-Aids for Young Scientists (A) and for Challenging Exploratory Research of the Japan Society for the Promotion of Science. The study was also conducted as part of the activities of the Strategic Research Program for Brain Sciences of the Japan Agency of Medical Research and Development.

\section{References}

[1] Yasumura, A., Kokubo. N., Yamamoto, H., Nakagawa, E., Kaga, M., Hikari, K., et al. (2014) Neurobehavioral and Hemodynamic Evaluation of STROOP and Reverse STROOP Interference in Children with Attention-Deficit/Hyperactivity Disorder. Brain Development, 36, 97-106. http://dx.doi.org/10.1016/j.braindev.2013.01.005

[2] Kahneman, D. and Tversky, A. (1979) Prospect Theory: An Analysis of Decisions under Risk. Econometrica, 47, 263-292. http://dx.doi.org/10.2307/1914185

[3] Japanese WISC-III Publishing Committee (1998) Japanese Wechsler Intelligence Scale for Children-III. Nihon Bunka Kagakusha, Tokyo (in Japanese).

[4] Ueno, K., Kaizu, A. and Hattori, M. (2005) Psychological Assessment of Individuals with Mild Developmental Disorder. Nihon Bunka Kagakusha, Tokyo (in Japanese).

[5] Era, S., Tanaka, E. and Baba, H. (2011) Characteristics in WISC-III Profiles of Children with Writing Difficulty by Content of the Main Complaint. Japanese Journal on Support Systems for Developmental Disabilities, 10, 19-26 (in Japanese).

[6] American Psychiatric Association (2000) Diagnostic and Statistical Manual of Mental Disorders. 4th Edition, Text Rev., American Psychiatric Publishing, Washington DC.

[7] Joffe, S., Cook, E.F., Cleary, P.D., Clark, J.W. and Weeks, J.C. (2001) Quality of Informed Consent: A New Measure of Understanding among Research Subjects. Journal of the National Cancer Institute, 93, 139-147. http://dx.doi.org/10.1093/jnci/93.2.139

[8] Stunkel, L., Benson, M., McLellan, L., Sinaii, N., Bedarida, G., Emanuel, E., et al. (2010) Comprehension and Informed Consent: Assessing the Effect of a Short Consent Form. IRB, 32, 1-9.

[9] Ashiya, M., Doi, T. and Tomoda, K. (2013) Analysis of Intelligence in Children with Functional Hearing Loss Accompanied by Inattention Problems. Japan Journal of Logopedics and Phoniatrics, 54, 245-250 (in Japanese). http://dx.doi.org/10.5112/jjlp.54.245

[10] Ohta, M. (1987) Cognitive Disorders of Infantile Autism: A Study Employing the WISC, Spatial Relationship Conceptualization, and Gesture Imitations. Journal of Autism and Developmental Disorders, 17, 45-62. http://dx.doi.org/10.1007/BF01487259

[11] Oyama, T. and Hirosawa, A. (2007) A Study of Psychological Assessment about Children with High-Functioning Pervasive Developmental Disorder. Bulletin of Comprehensive Center for Education Practice of the Aichi University of Education, 10, 15-24 (in Japanese). 